\title{
A "NATURAL" NORM FOR THE METHOD OF CHARACTERISTICS USING DISCONTINUOUS FINITE ELEMENTS: 2D AND 3D CASE
}

\author{
JACQUes Baranger $^{1}$ And Ahmed Machmoum ${ }^{2}$
}

\begin{abstract}
We consider the numerical approximation of a first order stationary hyperbolic equation by the method of characteristics with pseudo time step $k$ using discontinuous finite elements on a mesh $\mathcal{T}_{h}$. For this method, we exhibit a "natural" norm \|\|$_{h, k}$ for which we show that the discrete variational problem $P_{h}^{k}$ is well posed and we obtain an error estimate. We show that when $k$ goes to zero problem $\left(P_{h}^{k}\right)$ (resp. the \|\|$_{h, k}$ norm) has as a limit problem $\left(P_{h}\right)$ (resp. the \|\|$_{h}$ norm) associated to the Galerkin discontinuous method. This extends to two and three space dimension our previous results obtained in one space dimension.

Résumé. On considère l'approximation numérique d'une équation hyperbolique stationnaire du premier ordre par la méthode des caractéristiques de pseudo pas de temps $k$ et des éléments finis discontinus sur un maillage $\mathcal{T}_{h}$. On construit, pour cette méthode, une norme "naturelle", notée \|\|$_{h, k}$, pour laquelle on montre que le problème variationnel approché $\left(P_{h}^{k}\right)$ est bien posé et on obtient une majoration d'erreur. On montre que, quand $k$ tend vers zéro, le problème $\left(P_{h}^{k}\right)$ (resp. la norme \|\|$_{h, k}$ ) a pour limite le problème $\left(P_{h}\right)$ (resp. la norme \|\|$_{h}$ ), associé à la méthode de Galerkin discontinue. Ceci étend à la dimension d'espace deux et trois nos résultats antérieurs obtenus en dimension un d'espace.
\end{abstract}

AMS Subject Classification. 65N30, 65N25.

Received: December 4, 1998. Revised: May 18, 1999.

\section{INTRODUCTION}

We consider the numerical approximation by discontinuous finite elements method of a stationary hyperbolic equation of first order. Then one can use the Galerkin discontinuous method of [9] or the method of characteristics with pseudo time step $k$ introduced in [4] for convection diffusion problems. The purpose of this paper is to study the links between these two methods.

In [5] the authors remark that on a triangular mesh when $k$ goes to zero the method of characteristics converges formally to the Galerkin discontinuous method. We have constructed in [1] a norm \|\|$_{h, k}$, dependent of $k$ and the mesh size $h$, and obtained error estimates in this norm. Moreover when $k$ goes to zero this norm tends to

Keywords and phrases. Method of characteristics, discontinuous finite elements, advection equation.

1 MCS, Université Lyon 1, 43 bd. du 11 Novembre 1918, 69622 Villeurbanne Cedex, France.

e-mail: baranger@lan.univ-lyon1.fr

2 Équipe de Modélisation, Équations aux Dérivées Partielles et Analyse Numérique, Université Hassan 2, Faculté des sciences et techniques Mohammedia, B.P 146, 20650 Morocco. e-mail: machmoum@deneb.uh2m.ac.ma 
the norm associated to the Galerkin discontinuous method:

$$
\|v\|_{h}=\left(|v|_{0, \Omega}^{2}+\int_{\Gamma_{h}}\left(v^{+}-v^{-}\right)^{2}|b \cdot n| \mathrm{d} \gamma+\int_{\Gamma^{-}} v^{2}|b \cdot n| \mathrm{d} \gamma\right)^{\frac{1}{2}} .
$$

For these reasons the norm \|\|$_{h, k}$ appears to be a "natural" norm for the study of the characteristics method.

But our result in [1] was limited to one dimensional problems. The purpose of this paper is to extend this result to two and three dimensional problems.

This study of the method of characteristics is motivated by the numerical simulation of viscoelastic fluid flows. In the differential models the viscoelastic part of the stress tensor satisfies a non linear transport equation. Numerical experiments with these models using the method of characteristics in a finite element context are reported in $[3,6]$. They show the same efficiency as other upwinding methods like discontinuous Galerkin or SUPG. A (limited) numerical analysis of a differential viscoelastic model is made in [2]. In our opinion a good knowledge of the method of characteristics is compulsory for the numerical analysis of integral viscoelastic models. Such a study remains an open problem.

The paper is organised as follows: in Section 2 we recall a few facts about characteristics and Galerkin discontinuous method; in Section 3 we introduce a $k$ dependent variational formulation of the method of characteristics based on discontinuous $P_{r}$ finite elements with bilinear form $B_{c}$ and linear form $L_{c}$ and the natural norm \|\|$_{h, k}$ and show their convergence to respectively $B, L$ and \|\|$_{h}$ associated to the Galerkin discontinuous method; in Section 4 we study the coercivity of $B_{c}$ with respect to the norm \|\|$_{h, k}$ and obtain an error estimate $\left\|u-u_{h}\right\|_{h, k}=O\left(h^{r+1} / \sqrt{k}+h^{r+1}+k\right)$. These result are proved thanks to a "thick" Green formula.

\section{Discontinuous Galerkin method And CHARACteristics Method}

\subsection{Discontinuous Galerkin method}

We consider the scalar problem

$$
(P) \begin{cases}b \cdot \nabla u+c u=f & \text { in } \Omega \\ u=g_{1} & \text { on } \Gamma^{-}\end{cases}
$$

with $\Gamma^{-}=\{x \in \Gamma, \quad b \cdot n(x)<0\}$, where $n$ designate the unit outward normal to $\Gamma$. We suppose that $g_{1} \in H^{\frac{1}{2}}\left(\Gamma^{-}\right)$; then there exists, $g \in H^{1}(\Omega)$ such that $g=g_{1}$ on $\Gamma^{-}$.

In order to simplify the presentation $\Omega \subset \mathbb{R}^{2}$ is supposed to be polygonal and is equipped with uniformly regular triangulation $\mathcal{T}_{h}$ made of triangles $K$ :

$$
\bar{\Omega}=\bigcup_{K \in \mathcal{T}_{h}} K
$$

(Extension to 3D is easy.)

We denote by $\Gamma_{h}$ the inner sides of the mesh. We suppose that the mesh is compatible with $\Gamma^{-}$. For a function $u$, continuous in each $K$, but discontinuous on $\Gamma_{h}$ we define $u^{+}$and $u^{-}$by

$$
u^{+}(x)=\lim _{\varepsilon \rightarrow 0^{+}} u(x+\varepsilon b), \quad u^{-}(x)=\lim _{\varepsilon \rightarrow 0^{+}} u(x-\varepsilon b)
$$

and the jump of $u$ by

$$
[u]=u^{+}-u^{-}
$$


Let $u_{h} \in V_{h}=\left\{u_{h} \in L^{2}(\Omega) ;\left.\quad u_{h}\right|_{K} \in P_{r}\right\}$, where $P_{r}$ is the set of polynomials of degree less or equal to $r$ on $K \in \mathcal{T}_{h}$. The discontinuous Galerkin method for $(P)$ is defined in [9] by

$$
\int_{K}\left(b \cdot \nabla u_{h}+c u_{h}\right) v_{h} \mathrm{~d} x+\int_{\partial K^{-}}\left[u_{h}\right] v_{h}|n \cdot b| \mathrm{d} \sigma=\int_{K} f v_{h} \mathrm{~d} x \quad \forall v_{h} \in P_{r}(K)
$$

with the convention $\quad u_{h}^{-}=g$ if $\partial K^{-} \subset \Gamma^{-}$, where

$$
\partial K^{-}=\{x \in \partial K, \quad b \cdot n(x)<0\} \text { and } \partial K^{+}=\{x \in \partial K, \quad b \cdot n(x)<0\} .
$$

Setting

$$
\begin{aligned}
B\left(u_{h}, v_{h}\right) & =\sum_{K \in \mathcal{T}_{h}} \int_{K}\left(b \cdot \nabla u_{h}+c u_{h}\right) v_{h} \mathrm{~d} x+\sum_{\gamma \in \Gamma_{h}} \int_{\gamma}\left[u_{h}\right] v_{h}^{+}|n \cdot b| \mathrm{d} \sigma+\int_{\Gamma^{-}} u_{h} v_{h}|n \cdot b| \mathrm{d} \sigma \\
L\left(v_{h}\right) & =\int_{\Omega} f v_{h} \mathrm{~d} x+\int_{\Gamma^{-}} g_{1} v_{h}|n \cdot b| \mathrm{d} \sigma
\end{aligned}
$$

we can write (2.1) in variational form:

$$
\left(P_{h}\right) \quad B\left(u_{h}, v_{h}\right)=L\left(v_{h}\right) \quad \forall v_{h} \in V_{h} .
$$

Introducing the associate norm

$$
\left\|u_{h}\right\|_{h}^{2}=\left|u_{h}\right|_{0, \Omega}^{2}+\int_{\Gamma_{h}}\left[u_{h}\right]^{2}|n \cdot b| \mathrm{d} \sigma+\int_{\Gamma^{-}} u_{h}^{2}|n \cdot b| \mathrm{d} \sigma
$$

the following error estimate is shown in [8]:

Theorem 2.1. Suppose that $b \in W^{1, \infty}(\Omega)$, there exists a positive constant $c_{0}$ such that $c-\frac{1}{2} \nabla \cdot b \geq c_{0}>0$ and $u \in H^{r+1}(\Omega)$; then we have

$$
\left\|u-u_{h}\right\|_{h}=O\left(h^{r+\frac{1}{2}}\right) .
$$

An example presented in [11] shows that this estimation can't be improved on a quasiuniform mesh.

We recall that the coercivity of $B$ can be obtained by the following calculation that is used as a model in Section 3 in order to study the coercivity of $B_{c}$.

Theorem 2.2. Under the hypothesis $c-\frac{1}{2} \nabla \cdot b \geq c_{0}>0$ we have

$$
B(u, u) \geq C\|u\|_{h}^{2} .
$$

Proof. We have

$$
\int_{K}((b \cdot \nabla u) v+(b \cdot \nabla v) u+(\nabla \cdot b) u v) \mathrm{d} x=\int_{\partial K} u v b \cdot n \mathrm{~d} \sigma .
$$

Then we obtain

$$
\begin{aligned}
B(u, v)= & \int_{\Omega}\left(c-\frac{1}{2} \nabla \cdot b\right) u^{2} \mathrm{~d} x+\sum_{K} \int_{\partial K} \frac{1}{2} u^{2} b \cdot n \mathrm{~d} \sigma \\
& +\sum_{\gamma \subset \Gamma_{h}} \int_{\gamma}\left(u^{+}-u^{-}\right) u^{+}|n \cdot b| \mathrm{d} \sigma+\sum_{\gamma \subset \Gamma} \int_{\gamma \cap \Gamma^{-}}\left(u^{-}\right)^{2}|n \cdot b| \mathrm{d} \sigma .
\end{aligned}
$$


But we have (see [9]):

$$
\begin{aligned}
\sum_{K \in \mathcal{T}_{h}} \int_{\partial K} u^{2} b \cdot n \mathrm{~d} \sigma= & \sum_{\gamma \subset \Gamma_{h}} \int_{\gamma}\left(\left(u^{-}\right)^{2}-\left(u^{+}\right)^{2}\right)|n \cdot b| \mathrm{d} \sigma+\sum_{\gamma \subset \Gamma} \int_{\gamma \cap \Gamma^{-}}-\left(u^{+}\right)^{2}|n \cdot b| \mathrm{d} \sigma \\
& \left.+\int_{\gamma \cap \Gamma^{+}}\left(u^{-}\right)^{2}|n \cdot b| \mathrm{d} \sigma\right),
\end{aligned}
$$

and it follows that

$$
\begin{aligned}
B(u, u)= & \int_{\Omega}\left(c-\frac{1}{2} \nabla \cdot b\right) u^{2} \mathrm{~d} x+\sum_{\gamma \subset \Gamma_{h}} \int_{\gamma} \frac{1}{2}\left(\left(u^{-}\right)^{2}-\left(u^{+}\right)^{2}+2\left(u^{+}-u^{-}\right) u^{+}\right)|n \cdot b| \mathrm{d} \sigma \\
& +\frac{1}{2} \int_{\Gamma} u^{2}|n \cdot b| \mathrm{d} \sigma .
\end{aligned}
$$

Then we obtain

$$
B(u, u) \geq C\|u\|_{h}^{2},
$$

where $C=\min \left(c_{0}, 1 / 2\right)$.

\subsection{Characteristics method}

We introduce a parameter $k$ (pseudo time step) and we denote by $S(x, t, \tau)$ the solution of differential system of characteristics:

$$
\left\{\begin{array}{l}
\frac{\mathrm{d} S}{\mathrm{~d} \tau}=b(S) \\
S(x, t, t)=x
\end{array}\right.
$$

We define

$$
\begin{gathered}
X^{k}(x)=S(x, t, t-k) \\
\theta^{k}(x)=S(x, t, t+k) .
\end{gathered}
$$

Remark 2.1. We have $\theta^{k}\left(X^{k}(x)\right)=X^{k}\left(\theta^{k}(x)\right)=x$.

Following [4], we approximate the problem $(P)$ by

$$
\left(P^{k}\right) \begin{cases}\frac{u^{k}(x)-u^{k}\left(X^{k}(x)\right)}{k}+c(x) u^{k}(x)=f(x) & \text { if } x \in \Omega \\ u^{k}(x)=g\left(\theta^{k}(x)\right) & \text { if } x \in X^{k}(\Omega) \backslash \Omega .\end{cases}
$$

Following [2] a variational formulation of problem $\left(P^{k}\right)$ is given by:

$$
\begin{aligned}
& \text { Find } u^{k} \in L^{2}(\Omega) \text { such that } \\
& \frac{1}{k}\left(u^{k}, \tau\right)-\frac{1}{k}\left(u^{k}\left(X^{k}(.)\right), \tau\right)_{0, \Omega_{1}}+\left(c u^{k}, \tau\right)=(f, \tau)+\frac{1}{k}(g, \tau)_{0, \Omega_{2}} \forall \tau \in L^{2}(\Omega)
\end{aligned}
$$

where $\Omega_{1}=\left\{x \in \Omega ; X^{k}(x) \in \Omega\right\}, \Omega_{2}=\left\{x \in \Omega ; X^{k}(x) \notin \Omega\right\}$ and $($,$) denote the L^{2}(\Omega)$ scalar product. 
Theorem 2.3. If $b \in W^{1, \infty}(\Omega), u \in W^{2, \infty}\left(X^{k}(\Omega) \cup \Omega\right)$ is a solution of $(P)$ satisfying

$$
u\left(X^{k}(x)\right)=g(x) \text { if } X^{k}(x) \notin \Omega
$$

and $u^{k}$ is a solution of $\left(P^{k}\right)$, then we have

$$
\left|u-u^{k}\right|_{0, \Omega} \leq C(b, u) k
$$

For the proof of Theorem 2.3 see [10] (this proof uses the techniques developed in [4] for a convection-diffusion problem).

\section{Link BetWeEn CHARACTERISTiCs Method AND DisCONTINUOUS GALERKin METHOD}

We introduce in this paragraph a natural norm well adapted to the study of characteristics method such that when $k$ goes to zero it reduces to the discontinuous Galerkin norm (1.1). In Section 5 an error estimate in this norm is given.

Let $V_{h}=\left\{v \in L^{2}(\Omega),\left.\quad v\right|_{K} \in P_{r}(K) \quad \forall K \in \mathcal{T}_{h}\right\}$. The discrete variational problem associated to (2.11) is then:

$$
\begin{aligned}
& \text { Find } u_{h}^{k} \in V_{h} \text { such that } \\
& \frac{1}{k}\left(u_{h}^{k}, \tau\right)-\frac{1}{k}\left(u_{h}^{k}\left(X^{k}(.)\right), \tau\right)_{0, \Omega_{1}}+\left(c u_{h}^{k}, \tau\right)=(f, \tau)+\frac{1}{k}(g, \tau)_{0, \Omega_{2}} \forall \tau \in V_{h} .
\end{aligned}
$$

More precisely we have

$$
\frac{1}{k} \int_{\Omega} u_{h} v_{h} \mathrm{~d} x-\frac{1}{k} \int_{\Omega_{1}} u_{h}\left(X^{k}(.)\right) v_{h}(x) \mathrm{d} x+\int_{\Omega} c u_{h} v_{h} \mathrm{~d} x=\int_{\Omega} f v_{h} \mathrm{~d} x+\frac{1}{k} \int_{\Omega_{2}} g v_{h} \mathrm{~d} x .
$$

The variational formulation (3.1) is then equivalent to

$$
\left(P_{h}^{k}\right) \quad B_{c}\left(u_{h}, v_{h}\right)=L_{c}\left(v_{h}\right) \quad \forall v_{h} \in V_{h}
$$

with

$$
\begin{aligned}
B_{c}\left(u_{h}, v_{h}\right) & =\frac{1}{k} \int_{\Omega} u_{h} v_{h} \mathrm{~d} x-\frac{1}{k} \int_{\Omega_{1}} u_{h}\left(X^{k}(.)\right) v_{h}(x) \mathrm{d} x+\int_{\Omega} c u_{h} v_{h} \mathrm{~d} x \\
L_{c}\left(v_{h}\right) & =\int_{\Omega} f v_{h} \mathrm{~d} x+\frac{1}{k} \int_{\Omega_{2}} g v_{h} \mathrm{~d} x .
\end{aligned}
$$

Let $K_{2}=\left\{x \in K ; X^{k}(x) \notin K\right\}$ be the set of points of $K$ that were not in $K$ at time $t-k$, and $K_{1}=K \backslash K_{2}$. 


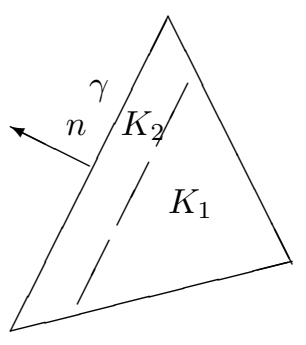

Figure 1.

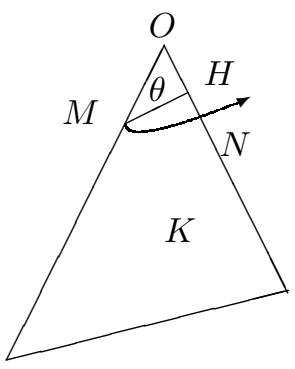

Figure 2 .

Then we have the situation shown in Figure 1.

We make the following hypothesis:

$(H 1) b$ is defined in a domain $D_{b}$ greater than $\Omega$ and $k$ is sufficiently small in order to have:

$$
\left\{X^{-t}(x), \quad x \in \partial K^{-} ; \quad 0 \leq t<k\right\} \subset D_{b}, \quad \forall K \in \mathcal{T}_{h} .
$$

(H2) CFL condition: $k$ is sufficiently small to insure that all $x \in K_{2} \subset K$ comes from a neighbouring triangle $K \prime$ of $K(K \prime \cap K \neq \emptyset)$.

(H3) $b$ and the mesh are such that: if $\gamma_{0}^{-}=\left\{x \in \gamma^{-} ; X^{-t}(x) \notin K \quad 0 \leq t<k\right\}$ then $m e s_{1-D} \gamma_{0}^{-} \leq C k \forall \gamma \subset \partial K$.

Remark 3.1. $H_{3}$ is technical hypothesis. Suppose that $\inf _{x \in D_{b}}|b(x)|=b_{i}>0$, then the hypothesis is verified in the two following situations:

1. Crossing characteristic: see Figure 2.

We denote by $\widehat{M N}$ the $\operatorname{arc}$ of $M$ to $N$ and $M N$ the segment of $M$ to $N$. We have

$$
\widehat{M N}=\int_{0}^{k}|s(t)| \mathrm{d} t \geq M N \inf _{x \in D_{b}}|b(x)|
$$

where $s(t)=b(x(t))$. We obtain:

$$
O M=\frac{M H}{\sin (\theta)} \leq \frac{M N}{\sin (\theta)} \leq \frac{b_{s}}{b_{i} \sin (\theta)} k
$$

where $b_{s}=\sup _{x \in D_{b}}|b(x)|$. Then we get

$$
\operatorname{mes}_{1-D} \gamma_{0}^{-}=O M \leq C k .
$$


2. Characteristic tangent to one side:

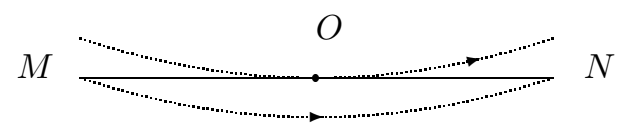

Figure 3.

We have

$$
\operatorname{mes}_{1-D} \gamma_{0}^{-}=O M \leq M N \leq \widehat{M N} \leq b_{s} k \leq C k
$$

This proves the Remark 3.1.

We define the natural norm \|\|$_{h, k}$ by

$$
\left\|u_{h}\right\|_{h, k}^{2}=\int_{\Omega} u_{h}^{2} \mathrm{~d} x+\frac{1}{k} \int_{\Omega_{2}} u_{h}^{2}(x) \mathrm{d} x+\frac{1}{k} \int_{\Omega_{1}}\left(u_{h}(x)-u_{h}\left(X^{k}(x)\right)\right)^{2} \mathrm{~d} x+\int_{\Omega_{\theta}} \frac{u_{h}^{2}\left(X^{k}(x)\right)}{k} \mathrm{~d} x
$$

where $\Omega_{\theta}=\theta^{k}(\Omega) \backslash \Omega$.

In the following result we will show that the discontinuous Galerkin method (2.4) is a limiting case of a characteristic method (3.2) introduced by [4] when applied to stationary convection problems and we show that the norm \|\|$_{h, k}$ defined by (3.5) tends to the norm \|\|$_{h}$ defined by (2.5) when $k$ goes to zero.

Theorem 3.1. Suppose that hypothesis $H_{1}, H_{2}, H_{3}$ are satisfied and that $b \in W^{1, \infty}\left(D_{b}\right)$ then the bilinear form $B_{c}$ and the linear form $L_{c}$ defined by the characteristics method converge respectively to the bilinear form $B$ and the linear form $L$ of the discontinuous Galerkin method when $k$ goes to zero; furthermore we have

$$
\lim _{k \rightarrow 0}\left\|u_{h}\right\|_{h, k}=\left\|u_{h}\right\|_{h}
$$

Proof. Using the discontinuous Galerkin method, the bilinear form $B$ is defined by

$$
B(u, v)=\sum_{K \in \mathcal{T}_{h}} \int_{K}(b \cdot \nabla u+c u) v \mathrm{~d} x+\sum_{\gamma \in \Gamma_{h}} \int_{\gamma}\left(u^{+}-u^{-}\right) v^{+}|n \cdot b| \mathrm{d} \sigma+\int_{\Gamma^{-}} u v|n \cdot b| \mathrm{d} \sigma .
$$

Using the characteristics method, the bilinear form $B_{c}$ is defined by

$$
B_{c}(u, v)=\frac{1}{k} \int_{\Omega} u v \mathrm{~d} x-\frac{1}{k} \int_{\Omega_{1}} u\left(X^{k}(x)\right) v(x) \mathrm{d} x+\int_{\Omega} c u v \mathrm{~d} x .
$$

In the sequel, we show that: for $u, v \in V_{h}$

$$
\lim _{k \rightarrow 0} B_{c}(u, v)=B(u, v) .
$$

We have

$$
\begin{aligned}
\lim _{k \rightarrow 0} \sum_{K \in \mathcal{T}_{h}} \int_{K} \frac{u(x)-u\left(X^{k}(x)\right)}{k} v \mathrm{~d} x= & \lim _{k \rightarrow 0} \sum_{K \in \mathcal{T}_{h}} \int_{K_{1}} \frac{u(x)-u\left(X^{k}(x)\right)}{k} v \mathrm{~d} x \\
& +\lim _{k \rightarrow 0} \sum_{K \in \mathcal{T}_{h}} \int_{K_{2}} \frac{u(x)-u\left(X^{k}(x)\right)}{k} v \mathrm{~d} x .
\end{aligned}
$$


On the one hand, the integral on $K_{1}$ becomes

$$
\begin{aligned}
\lim _{k \rightarrow 0} \int_{K_{1}} \frac{u(x)-u\left(X^{k}(x)\right)}{k} v(x) \mathrm{d} x & =\lim _{k \rightarrow 0} \int_{K_{1}} \frac{u(S(x, t, t))-u(S(x, t, t-k))}{k} v(x) \mathrm{d} x \\
& =\lim _{k \rightarrow 0} \int_{K_{1}}\left(\frac{\partial f}{\partial \tau}(t)+o(k)\right) v \mathrm{~d} x \quad(f(\tau)=u(S(x, t, \tau))) \\
& \left.=\lim _{k \rightarrow 0} \int_{K_{1}} b \cdot \nabla u+o(k)\right) v(x) \mathrm{d} x \\
& =\int_{K}(b \cdot \nabla u) v(x) \mathrm{d} x .
\end{aligned}
$$

On the other hand for the second term in the second member of (3.9) we now show that

$$
\lim _{k \rightarrow 0} \int_{\Gamma_{h}^{c}} \frac{u(x)-u\left(X^{k}(x)\right)}{k} v \mathrm{~d} x=\sum_{\gamma \in \Gamma_{h}} \int_{\gamma^{-}}\left(u^{+}-u^{-}\right) v^{+}|b \cdot n| \mathrm{d} \sigma,
$$

with $\Gamma_{h}^{c}=\left(\bigcup_{K \in \mathcal{T}_{h}} K_{2}\right) \backslash \Omega_{2}$.

Indeed, let $K_{2} \in \Gamma_{h}^{c}$ and $\gamma$ the common side of $K$ and $K^{\prime}\left(K, K^{\prime} \in \mathcal{T}_{h}\right)$, we have

$$
K_{2} \subset B^{-}=\left\{y=S(x, 0, t), x \in \gamma^{-} ; 0 \leq t<k\right\} .
$$

The points of $K_{2}$ are in a band $B^{-}$but this band contain other points which are not in $K_{2}$. We extend $u$ and $v$ which are a polynomials by the same expression in the whole band and we parameterise $\gamma^{-}(\gamma$ a side of $K)$ by $x=x^{0}+\sigma \tau, \tau$ being the unit vector of $\gamma$.

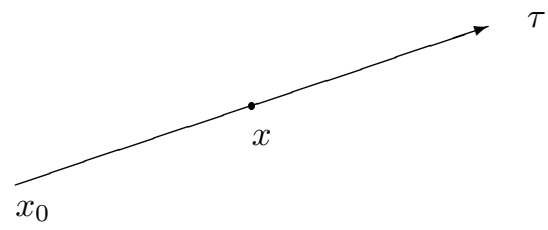

Figure 4 .

If we set $y(\sigma, t)=X^{-t}(x)=S\left(x^{0}+\sigma \tau, 0, t\right)$ then we have

$$
J(y(\sigma, t))=b \cdot n(S(x, 0, t))
$$

where $J(y(\sigma, t))$ is the Jacobian determinant with respect to $\sigma$ and $t$. Indeed,

$$
\begin{aligned}
J & =\left|\begin{array}{ll}
\frac{\partial y_{1}}{\partial \sigma} & \frac{\partial y_{1}}{\partial t} \\
\frac{\partial y_{2}}{\partial \sigma} & \frac{\partial y_{1}}{\partial t}
\end{array}\right| \\
& =\left|\begin{array}{ll}
\tau_{1} & b_{1}(S(x, 0, t)) \\
\tau_{2} & b_{2}(S(x, 0, t))
\end{array}\right| \\
& =\left(b_{2} \tau_{1}-b_{1} \tau_{2}\right)(S(x, 0, t)) \\
& =b \cdot n(S(x, 0, t)) .
\end{aligned}
$$


We have

$$
\begin{aligned}
\int_{\Gamma_{h}^{c}} \frac{u(x)-u\left(X^{k}(x)\right)}{k} v(x) \mathrm{d} x & =\sum_{K \in \mathcal{T}_{h}} \int_{K_{2}} \frac{u(x)-u\left(X^{k}(x)\right)}{k} v(x) \mathrm{d} x \\
& =\sum_{K \in \mathcal{T}_{h}} \sum_{\gamma \subset \partial K} \int_{K_{2} \cap B^{-}} \frac{u(x)-u\left(X^{k}(x)\right)}{k} v(x) \mathrm{d} x \\
& =\sum_{K \in \mathcal{T}_{h}} \sum_{\gamma \subset \partial K}\left(\int_{B^{-}} \frac{u(x)-u\left(X^{k}(x)\right)}{k} v(x) \mathrm{d} x\right. \\
& \left.-\int_{B^{-} \backslash\left(K_{2} \cap B^{-}\right)} \frac{u(x)-u\left(X^{k}(x)\right)}{k} v(x) \mathrm{d} x\right) \\
& =\sum_{K \in \mathcal{T}_{h}} \sum_{\gamma \subset \partial K}\left(\alpha_{1}-\alpha_{0}\right)
\end{aligned}
$$

with $B^{-}$the band associated to $\gamma^{-}$.

Let $x=x^{0}+\sigma \tau$ and $\delta$ the subset of $[0, k]$ corresponding to the $t$ such that $X^{-t}(x) \in K$. We have

$$
\begin{aligned}
\alpha_{0} & =\int_{B^{-} \backslash\left(K_{2} \cap B^{-}\right)} \frac{u(x)-u\left(X^{k}(x)\right)}{k} v(x) \mathrm{d} x \\
& =\int_{\gamma_{0}^{-}} \int_{\delta} \frac{u(y(\sigma, t))-u\left(X^{k}(y(\sigma, t))\right)}{k}|J(y(\sigma, t))| \mathrm{d} t \mathrm{~d} \sigma \\
& =\int_{\gamma_{0}^{-}} \int_{\delta} \phi(\sigma, t) \mathrm{d} t \mathrm{~d} \sigma
\end{aligned}
$$

where

$$
\phi(\sigma, t)=\frac{u(y(\sigma, t))-u\left(X^{k}(y(\sigma, t))\right)}{k}|J(y(\sigma, t))|
$$

and

$$
\begin{aligned}
y(\sigma, t) & =X^{-t}(x) \\
& =S(x, 0, t) \\
& =x+t b(x) \\
& =x^{0}+\sigma \tau+t b(x) .
\end{aligned}
$$

Using $H 3$ we obtain

$$
\begin{aligned}
\left|\alpha_{0}\right| & \leq\left|\gamma_{0}^{-}\right|\|\phi\|_{\infty} \quad\left(\phi \text { is continuous in } B^{-}\right) \\
& \leq C\|\phi\|_{\infty} k .
\end{aligned}
$$

Then we get: $\lim _{k \rightarrow 0} \alpha_{0}=0$. Moreover, setting

$$
\alpha_{1}=\int_{B^{-}} \frac{u(x)-u\left(X^{k}(x)\right)}{k} v(x) \mathrm{d} x=\frac{1}{k} \int_{\gamma^{-}} \int_{0}^{k} \phi(\sigma, t) \mathrm{d} t \mathrm{~d} \sigma
$$


we have

$$
\alpha_{1}-\int_{\gamma^{-}} \phi(\sigma, 0) \mathrm{d} \sigma=\frac{1}{k} \int_{\gamma^{-}} \int_{0}^{k}(\phi(\sigma, t)-\phi(\sigma, 0)) \mathrm{d} t \mathrm{~d} \sigma
$$

$\phi$ being uniformly continuous on $B^{-}$:

$$
\forall \epsilon>0 \quad \exists \eta \text { such that } \forall \sigma \in \gamma^{-} \forall t \in[0, \eta] \quad|\phi(\sigma, t)-\phi(\sigma, 0)| \leq \epsilon .
$$

So

$$
\left|\alpha_{1}-\int_{\gamma^{-}} \phi(\sigma, 0) \mathrm{d} \sigma\right| \leq\left|\gamma^{-}\right| \epsilon
$$

and we get

$$
\lim _{k \rightarrow 0} \alpha_{1}=\int_{\gamma^{-}} \phi(\sigma, 0) \mathrm{d} \sigma=\int_{\gamma^{-}}\left(u^{+}(x)-u^{-}(x)\right) v^{+}(x)|n \cdot b| \mathrm{d} x .
$$

From (3.14) we deduce

$$
\lim _{k \rightarrow 0} \int_{\Gamma_{h}^{c}} \frac{u(x)-u\left(X^{k}(x)\right)}{k} v(x) \mathrm{d} x=\sum_{K \in \mathcal{T}_{h}} \sum_{\gamma \subset \partial K} \int_{\gamma^{-}}\left(u^{+}-u^{-}\right) v^{+}|b \cdot n| \mathrm{d} x
$$

which proves (3.11).

From (3.8) we have

$$
B_{c}(u, v)=\frac{1}{k} \int_{\Omega_{2}} u v \mathrm{~d} x+\int_{\Omega_{1}} \frac{u(x)-u\left(X^{k}(x)\right)}{k} v(x) \mathrm{d} x+\int_{\Omega} c u v \mathrm{~d} x .
$$

Using the equality

$$
\Omega_{2}=\bigcup_{K \in \mathcal{A}} K_{2}
$$

where $\mathcal{A}$ is the union of triangles with at least one side in $\Gamma^{-}$, we get

$$
\int_{\Omega_{2}} \frac{u(x) v(x)}{k} \mathrm{~d} x=\sum_{K \in \mathcal{A}} \int_{K_{2}} \frac{u(x) v(x)}{k} \mathrm{~d} x .
$$

Using the same technique as above it follows that

$$
\begin{aligned}
\lim _{k \rightarrow 0} \int_{\Omega_{2}} \frac{u(x) v(x)}{k} \mathrm{~d} x & =\sum_{K \in \mathcal{A}} \int_{\gamma^{-}} u^{+} v^{+}|n \cdot b| \mathrm{d} \sigma \\
& =\int_{\Gamma^{-}} u^{+} v^{+}|n \cdot b| \mathrm{d} \sigma .
\end{aligned}
$$

From $K=K_{1} \cup K_{2}$ we have

$$
\Omega_{1}=\Gamma_{h}^{c} \cup\left\{K_{1} ; K \in \mathcal{T}_{h}\right\}
$$


Then we obtain

$$
\int_{\Omega_{1}} \frac{u(x)-u\left(X^{k}(x)\right)}{k} v(x) \mathrm{d} x=\int_{\Gamma_{h}^{c}} \frac{u(x)-u\left(X^{k}(x)\right)}{k} v(x) \mathrm{d} x+\sum_{K \in \mathcal{T}_{h}} \int_{K_{1}} \frac{u(x)-u\left(X^{k}(x)\right)}{k} v(x) \mathrm{d} x .
$$

Using (3.11) we get

$$
\lim _{k \rightarrow 0} \int_{\Gamma_{h}^{c}} \frac{u(x)-u\left(X^{k}(x)\right)}{k} v(x) \mathrm{d} x=\sum_{\gamma \in \Gamma_{h}} \int_{\gamma}\left(u^{+}-u^{-}\right) v^{+}|n \cdot b| \mathrm{d} \sigma
$$

and using (3.10) we obtain

$$
\lim _{k \rightarrow 0} \sum_{K \in \mathcal{T}_{h}} \int_{K_{1}} \frac{u(x)-u\left(X^{k}(x)\right)}{k} v(x) \mathrm{d} x=\sum_{K \in \mathcal{T}_{h}} \int_{K}(b \cdot \nabla u) v(x) \mathrm{d} x .
$$

From $(3.16,3.18,3.19)$ we conclude that

$$
\lim _{k \rightarrow 0} B_{c}(u, v)=B(u, v) .
$$

We now show that

$$
\lim _{k \rightarrow 0} L_{c}(v)=L(v)
$$

We have

$$
L_{c}(v)=\int_{\Omega} f v \mathrm{~d} x+\frac{1}{k} \int_{\Omega_{2}} g v \mathrm{~d} x
$$

Using the equality (3.15) and the method of proof of (3.11) we have

$$
\lim _{k \rightarrow 0} \frac{1}{k} \int_{\Omega_{2}} g v \mathrm{~d} x=\lim _{k \rightarrow 0} \frac{1}{k} \int_{\Omega_{2}} u\left(X^{k}(x)\right) v(x) \mathrm{d} x=\int_{\Gamma^{-}} u^{-} v^{+}|n \cdot b| \mathrm{d} \sigma=\int_{\Gamma^{-}} g v^{+}|n \cdot b| \mathrm{d} \sigma .
$$

This proves the result of convergence (3.20).

To conclude we prove that

$$
\lim _{k \rightarrow 0}\|u\|_{h, k}=\|u\|_{h}
$$

We recall that the norm \|\|$_{h, k}$ is defined by

$$
\|u\|_{h, k}^{2}=\int_{\Omega} u^{2}(x) \mathrm{d} x+\frac{1}{k} \int_{\Omega_{2}} u^{2}(x) \mathrm{d} x+\frac{1}{k} \int_{\Omega_{1}}\left(u(x)-u\left(X^{k}(x)\right)\right)^{2} \mathrm{~d} x+\int_{\Omega_{\theta}} \frac{u^{2}\left(X^{k}(x)\right)}{k} \mathrm{~d} x .
$$

From (3.16) we have the following result

$$
\lim _{k \rightarrow 0} \int_{\Omega_{2}} \frac{u^{2}(x)}{k} \mathrm{~d} x=\int_{\Gamma^{-}} u^{+2}|b \cdot n| \mathrm{d} \sigma .
$$

And using the equality

$$
\Omega_{\theta}=\left\{X^{-t}(x), x \in \Gamma^{+} ; 0 \leq t \leq k\right\}
$$


and the techniques of proof of (3.11), we have

$$
\lim _{k \rightarrow 0} \int_{\Omega_{\theta}} \frac{u^{2}\left(X^{k}(x)\right)}{k} \mathrm{~d} x=\int_{\Gamma^{+}} u^{-2}|b \cdot n| \mathrm{d} \sigma .
$$

Likewise, from (3.17) we get

$$
\frac{1}{k} \int_{\Omega_{1}}\left(u(x)-u\left(X^{k}(x)\right)\right)^{2} \mathrm{~d} x=\frac{1}{k} \int_{\Gamma_{h}^{c}}\left(u(x)-u\left(X^{k}(x)\right)\right)^{2} \mathrm{~d} x+\frac{1}{k} \sum_{K \in \mathcal{T}_{h}} \int_{K_{1}}\left(u(x)-u\left(X^{k}(x)\right)\right)^{2} \mathrm{~d} x .
$$

Like (3.11) we get

$$
\lim _{k \rightarrow 0} \frac{1}{k} \int_{\Gamma_{h}^{c}}\left(u(x)-u\left(X^{k}(x)\right)\right)^{2} \mathrm{~d} x=\sum_{\gamma \in \Gamma_{h}} \int_{\gamma}\left(u^{+}-u^{-}\right)^{2}|n \cdot b| \mathrm{d} \sigma
$$

and like (3.10):

$$
\lim _{k \rightarrow 0} \frac{1}{k} \int_{K_{1}}\left(u(x)-u\left(X^{k}(x)\right)\right)^{2} \mathrm{~d} x=\lim _{k \rightarrow 0} k \int_{K}(b \cdot \nabla u)^{2} \mathrm{~d} x=0 .
$$

From $(3.21,3.23-3.25)$ we conclude that

$$
\lim _{k \rightarrow 0}\|u\|_{h, k}=\|u\|_{h}
$$

This proves Theorem 3.1.

\section{Thick Green formula And Ellipticity of $B_{c}$}

We prove in this section that $B_{c}$ is elliptic in norm \|\|$_{h, k}$ under a suitable hypothesis. This result implies that problem $\left(P_{h}^{k}\right)$ as a unique solution. It is also used in the proof of the error estimate in Section 5 .

Ellipticity of $B_{c}$ is proved with the help of a "thick" Green formula which replace (2.7) for the method of characteristics.

Lemma 4.1 (thick Green formula).

$$
\begin{array}{r}
\frac{1}{k} \int_{\Omega_{2}} u(x) v(x) \mathrm{d} x+\int_{\Omega_{1}}\left(\frac{u(x)-u\left(X^{k}(x)\right)}{k} v(x)-\frac{v\left(X^{k}(x)\right)-v(x)}{k} u\left(X^{k}(x)\right)\right) \mathrm{d} x-\int_{\Omega_{\theta}} \frac{u\left(X^{k}(x)\right) v\left(X^{k}(x)\right)}{k} \mathrm{~d} x \\
=\int_{\Omega} \frac{1-\left|\frac{\mathrm{d} x}{\mathrm{~d} y}\right|}{k} u(y) v(y) \mathrm{d} y, \quad(4.1)
\end{array}
$$

where $\left|\frac{\mathrm{d} x}{\mathrm{~d} y}\right|=\left|\frac{\mathrm{d} \theta^{k}(y)}{\mathrm{d} y}\right|$ is the Jacobian. 
Proof. We have

$$
\begin{aligned}
& \frac{1}{k} \int_{\Omega_{2}} u(x) v(x) \mathrm{d} x+\int_{\Omega_{1}}\left(\frac{u(x)-u\left(X^{k}(x)\right)}{k} v(x)-\frac{v\left(X^{k}(x)\right)-v(x)}{k} u\left(X^{k}(x)\right)\right) \mathrm{d} x \\
&-\int_{\Omega_{\theta}} \frac{u\left(X^{k}(x)\right) v\left(X^{k}(x)\right)}{k} \mathrm{~d} x=\frac{1}{k} \int_{\Omega} u(x) v(x) \mathrm{d} x-\frac{1}{k} \int_{\Omega_{1} \cup \Omega_{\theta}} u\left(X^{k}(x)\right) v\left(X^{k}(x)\right) \mathrm{d} x \\
&=\int_{\Omega} \frac{1-\left|\frac{\mathrm{d} x}{\mathrm{~d} y}\right|}{k} u(y) v(y) \mathrm{d} y \quad\left(x=\theta^{k}(y) \text { and } X^{k}\left(\Omega_{1} \cup \Omega_{\theta}\right)=\Omega\right) .
\end{aligned}
$$

This proves (4.1).

Theorem 4.1. Suppose that there exist $c_{0}>0$ such that:

$$
c+\frac{1}{2} \frac{1-\left|\frac{\mathrm{d} x}{\mathrm{~d} y}\right|}{k} \geq c_{0}>0,
$$

where $x=\theta^{k}(y)$. Then we have

$$
B_{c}(u, u) \geq C\|u\|_{h, k}^{2}
$$

Proof. Applying (3.8) for $v=u$ we have

$$
B_{c}(u, u)=\int_{\Omega_{2}} \frac{u^{2}(x)}{k} \mathrm{~d} x+\int_{\Omega_{1}} \frac{u(x)-u\left(X^{k}(x)\right)}{k} u(x) \mathrm{d} x+\int_{\Omega} c(x) u^{2}(x) \mathrm{d} x,
$$

and for the previous Lemma with $v=u$ we obtain

$$
\begin{aligned}
2 \int_{\Omega_{1}} \frac{u(x)-u\left(X^{k}(x)\right)}{k} u(x) \mathrm{d} x= & \int_{\Omega_{1}} \frac{\left(u(x)-u\left(X^{k}(x)\right)\right)^{2}}{k} \mathrm{~d} x+\int_{\Omega_{\theta}} \frac{u^{2}\left(X^{k}(x)\right)}{k} \mathrm{~d} x \\
& -\int_{\Omega_{2}} \frac{u^{2}(x)}{k} \mathrm{~d} x+\int_{\Omega} \frac{1-\left|\frac{\mathrm{d} x}{\mathrm{~d} y}\right|}{k} u^{2}(y) \mathrm{d} y .
\end{aligned}
$$

Then we deduce that

$$
\begin{aligned}
B_{c}(u, u)= & \frac{1}{2 k} \int_{\Omega_{2}} u^{2}(x) \mathrm{d} x+\frac{1}{2 k} \int_{\Omega_{\theta}} u^{2}\left(X^{k}(x)\right) \mathrm{d} x+\frac{1}{2 k} \int_{\Omega_{1}}\left(u(x)-u\left(X^{k}(x)\right)\right)^{2} \mathrm{~d} x \\
& +\int_{\Omega}\left(c+\frac{1}{2 k}\left(1-\left|\frac{\mathrm{d} x}{\mathrm{~d} y}\right|\right)\right) u^{2}(y) \mathrm{d} y .
\end{aligned}
$$

Then if $c+\frac{1}{2 k}\left(1-\left|\frac{\mathrm{d} x}{\mathrm{~d} y}\right|\right) \geq c_{0}>0$, we have the coercivity of $B_{c}$ with respect to the norm \|\|$_{h, k}$.

Remark 4.1. We remark that we have the following convergency result:

$$
\lim _{k \rightarrow 0} \frac{1-\left|\frac{\mathrm{d} x}{\mathrm{~d} y}\right|}{k}=-\nabla \cdot b .
$$


Indeed, we have

$$
\begin{aligned}
x & =\theta^{k}(y)=y+k b(y)+\cdots \\
\frac{\mathrm{d} x}{\mathrm{~d} y} & =I+k \nabla b(y)+\cdots
\end{aligned}
$$

So

$$
\left|\frac{\mathrm{d} x}{\mathrm{~d} y}\right|=1+k \nabla \cdot b(y)+\cdots
$$

Then we conclude that

$$
\frac{1-\left|\frac{\mathrm{d} x}{\mathrm{~d} y}\right|}{k}=-\nabla \cdot b(y)+O(k)
$$

This establishes the result.

\section{ERror estimate}

To give an error estimate for the characteristics method we shall use another form of $B_{c}$ shown in the following Lemma and suggested by the second expression of $B$ of the discontinuous Galerkin method.

Lemma 5.1. The bilinear form $B_{c}$ can be written as

$$
B_{c}(u, v)=\int_{\Omega}\left(c(y)+\frac{1-\left|\frac{\mathrm{d} x}{\mathrm{~d} y}\right|}{k}\right) u(y) v(y) \mathrm{d} y-\int_{\Omega_{1}} \frac{v(x)-v\left(X^{k}(x)\right)}{k} u\left(X^{k}(x)\right) \mathrm{d} x+\int_{\Omega_{\theta}} \frac{u\left(X^{k}(x)\right) v\left(X^{k}(x)\right)}{k} \mathrm{~d} x .
$$

Proof. Applying the thick Green formula (4.1) to $\left(P_{h}^{k}\right)$ we get

$$
\begin{aligned}
\int_{\Omega}\left(c(y)+\frac{1-\left|\frac{\mathrm{d} x}{\mathrm{~d} y}\right|}{k}\right) u(y) v(y) \mathrm{d} y-\int_{\Omega_{1}} \frac{v(x)-v\left(X^{k}(x)\right)}{k} u\left(X^{k}(x)\right) \mathrm{d} x+ & \int_{\Omega_{\theta}} \frac{u\left(X^{k}(x)\right) v\left(X^{k}(x)\right)}{k} \mathrm{~d} x= \\
& \int_{\Omega} f(x) v(x) \mathrm{d} x+\frac{1}{k} \int_{\Omega_{2}} g(x) v(x) \mathrm{d} x .
\end{aligned}
$$

If we compare (5.2) with $\left(P_{h}^{k}\right)$ we deduce the result 5.1.

Lemma 5.2. If $b \in W^{1, \infty}(\Omega), u \in W^{2, \infty}\left(X^{k}(\Omega) \cup \Omega\right)$ is a solution of $(P)$ satisfying

$$
u\left(X^{k}(x)\right)=g(x) \text { if } X^{k}(x) \notin \Omega
$$

then we have the consistency relation

$$
B_{c}\left(u-u_{h}, v_{h}\right) \leq C k\left\|v_{h}\right\|_{h, k} \quad \forall v_{h} \in V_{h}
$$

where $u_{h}$ is the solution of $\left(P_{h}^{k}\right)$. 
Proof. Let $v_{h} \in V_{h}$, we have

$$
\begin{aligned}
B_{c}\left(u, v_{h}\right) & =\frac{1}{k} \int_{\Omega} u(x) v_{h}(x) \mathrm{d} x-\frac{1}{k} \int_{\Omega_{1}} u\left(X^{k}(x)\right) v_{h}(x) \mathrm{d} x+\int_{\Omega} c(x) u(x) v_{h}(x) \mathrm{d} x \\
& =\frac{1}{k} \int_{\Omega_{2}} u(x) v_{h}(x) \mathrm{d} x+\int_{\Omega_{1}} \frac{u(x)-u\left(X^{k}(x)\right)}{k} v_{h}(x) \mathrm{d} x+\int_{\Omega} c(x) u(x) v_{h}(x) \mathrm{d} x .
\end{aligned}
$$

So since $u$ is a solution of the continuous problem, we have

$$
b(x) \cdot \nabla u(x)+c(x) u(x)=f(x) .
$$

Then

$$
\begin{aligned}
B_{c}\left(u, v_{h}\right)= & \int_{\Omega_{2}}\left(\frac{u(x)}{k}-b(x) \cdot \nabla u(x)\right) v_{h}(x) \mathrm{d} x \\
& +\int_{\Omega_{1}}\left(\frac{u(x)-u\left(X^{k}(x)\right)}{k}-b(x) \cdot \nabla u(x)\right) v_{h}(x) \mathrm{d} x+\int_{\Omega} f(x) v_{h}(x) \mathrm{d} x .
\end{aligned}
$$

So since $u_{h}$ is a solution of problem $P_{h}^{k}$, we have

$$
B_{c}\left(u_{h}, v_{h}\right)=L_{c}\left(v_{h}\right)
$$

Then we obtain

$$
\begin{aligned}
B_{c}\left(u-u_{h}, v_{h}\right)= & \int_{\Omega_{2}}\left(\frac{u(x)-g(x)}{k}-b(x) \cdot \nabla u(x)\right) v_{h}(x) \mathrm{d} x \\
& +\int_{\Omega_{1}}\left(\frac{u(x)-u\left(X^{k}(x)\right)}{k}-b(x) \cdot \nabla u(x)\right) v_{h}(x) \mathrm{d} x,
\end{aligned}
$$

where $g(x)=u\left(\theta^{k}(x)\right)$.

On the one hand for the first term in the second member of (5.4) we have

$$
\begin{aligned}
\int_{\Omega_{2}}\left(\frac{u(x)-g(x)}{k}-b(x) \cdot \nabla u(x)\right) v_{h}(x) \mathrm{d} x & =\int_{\Omega_{2}}\left(\frac{1}{k} \int_{k}^{0} \frac{\mathrm{d}}{\mathrm{d} \tau} u \circ S(x, 0, \tau) \mathrm{d} \tau-b(x) \cdot \nabla u(x)\right) v_{h}(x) \mathrm{d} x \\
& \left.=\int_{\Omega_{2}} \frac{1}{k} \int_{k}^{0} b \cdot \nabla u(S(x, 0, \tau)) \mathrm{d} \tau-b(x) \cdot \nabla u(x)\right) v_{h}(x) \mathrm{d} x \\
& \leq C k|b|_{0, \infty}^{2}|u|_{1, \infty}\left|\Gamma^{-}\right|\left\|v_{h}\right\|_{h, k} \quad\left(\left|\Omega_{2}\right| \leq k|b|_{0, \infty}\left|\Gamma^{-}\right|\right) .
\end{aligned}
$$

On the other hand for the second term in the second member of (5.4) we have

$$
\int_{\Omega_{1}}\left(\frac{u(x)-u\left(X^{k}(x)\right)}{k}-b(x) \cdot \nabla u(x)\right) v_{h}(x) \mathrm{d} x=\frac{k}{2} \int_{\Omega_{1}} E(x) v_{h}(x) \mathrm{d} x
$$

where

$$
\begin{aligned}
\left(E, v_{h}\right)_{0, \Omega_{1}}= & \sum_{i, j} \int_{\Omega} b_{i}\left(\eta^{k}(x)\right) b_{j}\left(\eta^{k}(x)\right) \frac{\partial^{2} u}{\partial x_{i} \partial x_{j}}\left(\eta^{k}(x)\right) v_{h}(x) \mathrm{d} x \\
& +\sum_{i, j} \int_{\Omega} \frac{\partial u}{\partial x_{i}}\left(\eta^{k}(x)\right) \frac{\partial b_{i}}{\partial x_{j}}\left(\eta^{k}(x)\right) b_{j}\left(\eta^{k}(x)\right) v_{h}(x) \mathrm{d} x
\end{aligned}
$$


and $\eta^{k}(x)=S(x, k, \theta k)$ with $0<\theta<1$ (see [4]).

Then we get

$$
\int_{\Omega_{1}}\left(\frac{u(x)-u\left(X^{k}(x)\right)}{k}-b(x) \cdot \nabla u(x)\right) v_{h}(x) \mathrm{d} x \leq \frac{k}{2}\left(|u|_{2, \infty}|b|_{0, \infty}^{2}+|u|_{1, \infty}|b|_{1, \infty}|b|_{0, \infty}\right)\left\|v_{h}\right\|_{h, k} .
$$

Finally we deduce that we have

$$
B_{c}\left(u-u_{h}, v_{h}\right) \leq C k\left\|v_{h}\right\|_{h, k}
$$

This proves the Lemma 5.2.

\section{Error estimate}

Let $P_{h}$ be the $L^{2}(\Omega)$-orthogonal projection into $V_{h}$, then we have the following error estimate (see [7]):

Lemma 5.3. Let $m=0,1,1 \leq p \leq+\infty$ and $r \geq 0$. There exists a positive constant $C$ independent of $h$ such that

$$
\left|v-P_{h} v\right|_{m, p, K} \leq C h_{K}^{r+1-m}|v|_{r+1, p, K}, \quad \forall v \in W^{r+1, p}(K) .
$$

Then we have a priori error estimate of problem $(P 1)$ treated by the characteristics method.

Theorem 5.1. Suppose that $b \in W^{1, \infty}(\Omega), u \in W^{r+1, \infty}\left(X^{k}(\Omega) \cup \Omega\right)(r>0)$ is a solution of $(P)$ satisfying $u\left(X^{k}(x)\right)=g(x)$ if $X^{k}(x) \notin \Omega$ and there exists $c_{0}>0$ such that

$$
c+\frac{1}{2} \frac{1-\left|\frac{\mathrm{d} x}{\mathrm{~d} y}\right|}{k} \geq c_{0}>0
$$

where $x=\theta^{k}(y)$. Then we have

$$
\left\|u-u_{h}\right\|_{h, k}=O\left(h^{r+1}+\frac{h^{r+1}}{\sqrt{k}}+k\right) .
$$

Proof. Let $P_{h} u$ be the $L^{2}(\Omega)$-orthogonal projection of $u$ into $V_{h}$ we have

$$
c\left\|u_{h}-P_{h} u\right\|_{h, k}^{2} \leq B_{c}\left(u_{h}-P_{h} u, u_{h}-P_{h} u\right) \leq B_{c}\left(u-P_{h} u, u_{h}-P_{h} u\right)+C k\left\|u_{h}-P_{h} u\right\|_{h, k} .
$$

Under (5.1) we have

$$
\begin{aligned}
B_{c}\left(u-P_{h} u, u_{h}-P_{h} u\right)= & \int_{\Omega}\left(c(y)+\frac{1-\left|\frac{\mathrm{d} x}{\mathrm{~d} y}\right|}{k}\right)\left(u-P_{h} u\right)(y)\left(u_{h}-P_{h} u\right)(y) \mathrm{d} y \\
& -\int_{\Omega_{1}} \frac{\left(u_{h}-P_{h} u\right)(x)-\left(u_{h}-P_{h} u\right)\left(X^{k}(x)\right)}{k}\left(u-P_{h} u\right)\left(X^{k}(x)\right) \mathrm{d} x \\
& +\int_{\Omega_{\theta}} \frac{\left(u_{h}-P_{h} u\right)\left(X^{k}(x)\right)\left(u-P_{h} u\right)\left(X^{k}(x)\right)}{k} \mathrm{~d} x .
\end{aligned}
$$

If we denote $S$ by:

$$
S=\left(\frac{1}{k} \int_{\Omega_{1}}\left(u-P_{h} u\right)^{2}\left(X^{k}(x)\right) \mathrm{d} x\right)^{\frac{1}{2}}
$$


then we can estimate (5.9) by:

$$
\begin{aligned}
& \int_{\Omega_{1}} \frac{\left(u_{h}-P_{h} u\right)(x)-\left(u_{h}-P_{h} u\right)\left(X^{k}(x)\right)}{k}\left(u-P_{h} u\right)\left(X^{k}(x)\right) \mathrm{d} x \\
& \quad \leq\left(\frac{1}{k} \int_{\Omega_{1}}\left(\left(u_{h}-P_{h} u\right)(x)-\left(u_{h}-P_{h} u\right)\left(X^{k}(x)\right)\right)^{2} \mathrm{~d} x\right)^{\frac{1}{2}}\left(\frac{1}{k} \int_{\Omega_{1}}\left(u-P_{h} u\right)^{2}\left(X^{k}(x)\right) \mathrm{d} x\right)^{\frac{1}{2}} \leq\left\|u_{h}-P_{h} u\right\|_{h, k} S .
\end{aligned}
$$

Likewise, if we define $T$ by:

$$
T=\left(\int_{\Omega_{\theta}} \frac{\left(u-P_{h} u\right)^{2}\left(X^{k}(x)\right)}{k} \mathrm{~d} x\right)^{\frac{1}{2}}
$$

then we can estimate (5.10) by:

$$
\begin{aligned}
\int_{\Omega_{\theta}} \frac{\left(u_{h}-P_{h} u\right)\left(X^{k}(x)\right)\left(u-P_{h} u\right)\left(X^{k}(x)\right)}{k} \mathrm{~d} x & \leq\left(\int_{\Omega_{\theta}} \frac{\left(u_{h}-P_{h} u\right)^{2}\left(X^{k}(x)\right)}{k}\right)^{\frac{1}{2}}\left(\int_{\Omega_{\theta}} \frac{\left(u-P_{h} u\right)^{2}\left(X^{k}(x)\right)}{k}\right)^{\frac{1}{2}} \\
& \leq\left\|u_{h}-P_{h} u\right\|_{h, k} T .
\end{aligned}
$$

Then to estimate $\left\|u_{h}-P_{h} u\right\|_{h, k}$, one has only to estimate $S$ and $T$.

For $S$ we have

$$
\begin{aligned}
S=\left(\frac{1}{k} \int_{X^{k}\left(\Omega_{1}\right)}\left(u-P_{h} u\right)^{2}(y)\left|\frac{\mathrm{d} x}{\mathrm{~d} y}\right| \mathrm{d} y\right)^{\frac{1}{2}}\left(x=\theta^{k}(y)\right) & \leq\left(\frac{1}{k} \int_{\Omega}\left(u-P_{h} u\right)^{2}(x) \mathrm{d} x\right)^{\frac{1}{2}} \\
& \leq \sum_{K \in \mathcal{T}_{h}}\left(\frac{1}{k} \int_{K}\left(u-P_{h} u\right)^{2}(x) \mathrm{d} x\right)^{\frac{1}{2}} \\
& \leq \frac{1}{\sqrt{k}} \sum_{K \in \mathcal{T}_{h}}\left\|u-P_{h} u\right\|_{0,2, K} \\
& \leq \frac{C}{\sqrt{k}} h_{K}^{r+1}|u|_{r+1,2, K} .
\end{aligned}
$$

For $T$ we have

$$
\begin{aligned}
T=\left(\frac{1}{k} \int_{X^{k}\left(\Omega_{\theta}\right)}\left(u-P_{h} u\right)^{2}(y)\left|\frac{\mathrm{d} x}{\mathrm{~d} y}\right| \mathrm{d} y\right)^{\frac{1}{2}}\left(x=\theta^{k}(y)\right) & \leq\left(\frac{1}{k} \int_{\Omega}\left(u-P_{h} u\right)^{2}(x) \mathrm{d} x\right)^{\frac{1}{2}} \\
& \leq \frac{1}{\sqrt{k}} \sum_{K \in \mathcal{T}_{h}}\left\|u-P_{h} u\right\|_{0,2, K} \\
& \leq \frac{C}{\sqrt{k}} h_{K}^{r+1}|u|_{r+1,2, K} .
\end{aligned}
$$

Further, according to the definition of the norm \|\|$_{h, k}$ we have

$$
\begin{aligned}
\left\|P_{h} u-u\right\|_{h, k} \leq & C\left(\left|P_{h} u-u\right|_{0, \Omega}+\frac{1}{\sqrt{k}}\left|P_{h} u-u\right|_{0, \Omega}\right)+\left(\frac{1}{k} \int_{\Omega_{1}}\left(u-P_{h} u\right)^{2}\left(X^{k}(x)\right) \mathrm{d} x\right)^{\frac{1}{2}} \\
& +\left(\int_{\Omega_{\theta}} \frac{\left(u-P_{h} u\right)^{2}\left(X^{k}(x)\right)}{k} \mathrm{~d} x\right)^{\frac{1}{2}} .
\end{aligned}
$$


Then we conclude that $\left\|P_{h} u-u\right\|_{h, k}$ can be also estimated using $S$ and $T$.

Finally we have

$$
\left\|u_{h}-u\right\|_{h, k} \leq\left\|u_{h}-P_{h} u\right\|_{h, k}+\left\|P_{h} u-u\right\|_{h, k} .
$$

Then we obtain

$$
\left\|u-u_{h}\right\|_{h, k}=O\left(h^{r+1}+\frac{h^{r+1}}{\sqrt{k}}\right) .
$$

This proves the Theorem 4.1.

Remark 5.1. For $k \leq h$, we can remark that the error estimate is at best $O\left(h^{r+\frac{1}{2}}\right)$, like the error estimate given by the discontinuous Galerkin method [8] and is exactly $O\left(h^{r+\frac{1}{2}}\right)$ for $k=C h$.

\section{REFERENCES}

[1] J. Baranger and A. Machmoum, Une norme naturelle pour la méthode des caractéristiques en éléments finis discontinus : cas 1-D. RAIRO Modél. Math. Anal. Numér. 30 (1996) 549-574.

[2] J. Baranger and A. Machmoum, Exitence of approximate solutions and error bounds for viscoelastic fluid flow: Characteristics method. Comput. Methods Appl. Mech. Engrg. 148 (1997) 39-52.

[3] F. Basombrio, Flows in viscoelastic fluids treated by the method of characteristics. J. Non-Newtonian Fluid Mech. 39 (1991) $17-34$.

[4] A. Bermudez and J. Durany, La méthode des caractéristiques pour les problèmes de convection-diffusion stationnaires. RAIRO Modél. Math. Anal. Numér. 21 (1987) 7-26.

[5] M. Fortin and A. Fortin, Une note sur les méthodes de caractéristiques et de Lesaint-Raviart pour les problèmes hyperboliques stationnaires. RAIRO Modél. Math. Anal. Numér. 23 (1989) 593-596.

[6] M. Fortin and D. Esselaoui, A finite element procedure for viscoelastic flows. Int. J. Numer. Methods Fluids 7 (1987) 1035-1052.

[7] V. Girault and P.A. Raviart, Finite element method for Navier Stokes equations, Theory and Algorithms. Springer, BerlinHeidelberg-New York (1986).

[8] C. Johnson and J. Pitkaranta, An analysis of the discontinuous Galerkin method for a scalar hyperbolic equation. Math. Comp. 46 (1987) 1-26.

[9] P. Lesaint and P.A. Raviart, On a finite element method for solving the neutron transport equations, in Mathematical aspects of finite element in partial differential equations, C. de Boor Ed., Academic Press (1974) 89-123.

[10] A. Machmoum, Méthodes numériques pour les fluides viscoélastiques : méthode des caractéristiques pour les modèles différentiels et intégraux. Thèse, Université Lyon 1, Laboratoire d'analyse numérique (1996).

[11] T.E. Paterson, A note on the convergence of the discontinuous Galerkin method for a scalar hyperbolic equation. SIAM $J$. Numer. Anal. 26 (1991) 133-140. 